Juan Pablo Herrera Saavedra*

Dennis Sánchez Navarro***

Superintendencia de Industria y Comercio Bogotá, Colombia.

Recibido: 20 de febrero de 2015

Concepto de evaluación: 7 de abril de 2015

Aprobado: 9 de diciembre de 2015

Artículo de reflexión

(C) 2016 Universidad Católica de Colombia.

Facultad de Ciencias

Económicas y Administrativas.

Todos los derechos reservados
* Máster en Teoría y Política Económica. Coordinador de Estudios Económicos de la Superintendencia de Industria y Comercio (SIC). Profesor de la Facultad de Ciencias Económicas de la Universidad Nacional de Colombia y de la Facultad de Economía de la Universidad Externado de Colombia, Bogotá, Colombia. Dirección de correspondencia: Tr. 1 este No. 65c-41 apt. 701, Bogotá, Colombia. Correos electrónicos:.jpherreras@unal.edu.co, jpherrera@sic.gov.co

** Economista y magíster en Economía.

Exfuncionaria del Departamento Nacional de Planeación y de la

Superintendencia de Industria y Comercio, Bogotá, Colombia. Dirección de correspondencia: Cra. 78 f No 0-33 Bloque 4 Int 17 Apt 102, Bogotá, Colombia.. Correo electrónico: dennis.seni@gmail.com
Finanz. polit. econ., ISSN: 2248-6046, Vol. 8, No. 1, enero-junio, 2016, pp. 157-164

http://dx.doi.org/10.14718/revfinanzpolitecon.2016.8.1.8

\section{Efectos de integraciones de firmas en mercados de bienes homogéneos"}

\section{RESUMEN}

Este artículo modela los posibles efectos que, ante una integración empresarial, se pueden producir sobre el precio de un mercado de un bien homogéneo. El análisis se realiza a partir de dos escenarios: a) considerando tecnologías similares entre firmas y b) asumiendo algún grado de heterogeneidad por grupos de empresas. Se encuentra que aun en presencia de operaciones en favor de la eficiencia en la oferta del producto, existen riesgos de incremento en precios.

Palabras clave: oligopolio, Cournot, bienes homogéneos, comportamiento de las firmas, producción, juegos no cooperativos.

JEL: D43, D21, D24, C72

\section{The effects of firm mergers on homogenous goods markets}

\section{ABSTRACT}

This article models the potential effects of firm mergers on the price of a market for homogenous goods. The analysis is carried out according to two scenarios: a) firms have similar levels of technology at their disposal and b) a degree of heterogeneity is assumed between groups of firms. It is found that even when mechanisms are in place to favor efficiency in product supply, there is a risk that prices will increase.

Keywords: Oligopoly, Cournot, homogenous goods, firm behavior, production, non-cooperative games.

\footnotetext{
* Los autores extienden un agradecimiento especial a Camila Trilleras, estudiante de la Universidad Externado de Colombia, y al profesor Jorge Iván González, de la misma universidad, por los valiosos comentarios y aportes al documento. De igual manera, especial agradecimiento a los evaluadores y al editor de la revista, por sus aportes.
} 


\section{Efeitos de integrações de empresas em mercados de bens homogêneos}

\section{RESUMO}

Este artigo modela os possíveis efeitos que, diante de uma integração empresarial, podem ser produzidos sobre o preço de um mercado de um bem homogêneo. A análise realiza-se a partir de dois cenários: a) ao considerar tecnologias similares entre empresas e b) ao assumir algum grau de heterogeneidade por grupos de empresas. Encontra-se que, ainda na presença de operações em favor da eficiência na oferta do produto, existem riscos de aumento em preços.

Palavras-chave: oligopólio, Cournot, bens homogêneos, comportamentos das empresas, produção, jogos não cooperativos. 


\section{INTRODUCCIÓN}

Desde el trabajo de Cournot (1838) y Bertrand (1883) se abre una agenda de discusión del más alto interés para la teoría económica: el papel de las interacciones entre firmas que determinan diferentes estructuras de mercado. Los esfuerzos de modelación se hicieron progresivamente más visibles durante los albores del siglo XX, a partir de trabajos como el de Chamberlin (1933) y von Stackelberg (1934), quienes refuerzan esta tendencia de modelación.

A su vez, los diálogos entre el derecho y la economía, a propósito del análisis económico en materia de protección de la competencia en las diferentes jurisdicciones en el mundo, permiten reconocer una tendencia creciente a utilizar este tipo de modelos como referencia para identificar los posibles efectos, en caso de llevarse a cabo una operación de integración empresarial con efectos horizontales, desde la óptica del riesgo de conductas unilaterales, principalmente, $y$, en algunos casos, de conductas coordinadas. Durante la década de los noventa, trabajos como los de Shapiro (1996), Werden (1997) y Epstein y Rubinfeld (2001), relacionados con simulación de integraciones empresariales, han dado cuenta de esta tendencia.

Por su parte, trabajos como el de Ding, Fan y Wolfstettter (2013) muestran un mecanismo de competencia, a la Cournot, para entender cómo diferentes integraciones de tipo horizontal, entre firmas que participan en distintas subastas, pueden generar sinergias que permiten obtener mayores beneficios.

Tsitsikilis y Xu (2014) proponen un análisis de bienestar social mediante una versión modificada del modelo de Cournot, desde una función de demanda de mercado convexa. Encontraron allí unos niveles mínimos de eficiencia en distintos escenarios de interacción dentro del mercado. A su vez, Beckman, DeAngelo y Smith (2012) proponen un modelo experimental para verificar la forma como las firmas tienden a posicionarse en el mercado. Encuentran que, sin importar el tamaño de las firmas, estas tienden a compartir los beneficios que un monopolista pudiese percibir en el mercado. En su simulación utilizan mecanismos de competencia a la Cournot y distintos escenarios de integraciones entre firmas para identificar los principales resultados.

Para el análisis de integraciones de tipo horizontal, García y Trillas (2011) analizan el impacto de las ofertas públicas de adquisición (OPA) a Endesa, Hidrocantábrico y Scottish Power sobre su valor medido por el precio en Bolsa. Los autores estudian diferentes investigadores que asumen que una integración empresarial "es una oportunidad para la empresa compradora, dada la posibilidad de crear valor, a pesar de los costos en que debe incurrir". Por su parte, Eckbo (1983) sostiene:

Las fusiones horizontales generan rendimientos anormales positivos a los accionistas de las empresas compradas porque aumenta la probabilidad de colusión entre productores rivales, y señala que la fusión aumenta el valor comercial de las empresas, vía eficiencia productiva, debido a una política de costos de producción más eficiente y a la reducción de los costos de transacción.

Otros autores citados en el documento referido realizan un estudio sobre fusiones y adquisiciones de empresas estadounidenses, entre 1973 y 1998, y encuentran que los propietarios de acciones de la empresa objeto de compra resultan beneficiados con la integración, pues la creación de valor para la empresa integrada llega en promedio al 16\%. En el mismo sentido, Eckbo (1983) presenta el beneficio como el objetivo que los rivales pueden esperar de la noticia de una fusión horizontal, que reduce significativamente los costes de la aplicación de un acuerdo colusorio tácito dentro de la industria de las empresas que se fusionan.

Un resultado importante del análisis es que los rivales de las 65 fusiones horizontales en la base de datos ganan rendimientos anormalmente 
positivos en el momento de la fusión. Un segundo resultado es el rendimiento de los rivales en el momento de la denuncia antimonopolio, para ser estadísticamente independiente de la actuación correspondiente en torno a la propuesta de fusión, lo que contradice la hipótesis de la colusión.

En esta línea argumental, el presente artículo pretende señalar un método de detección del posible efecto de explotación sobre consumidores derivado de integraciones empresariales, cuando se trata de mercados oligopólicos en los que los consumidores perciben bienes homogéneos. Para el efecto, se abordará el problema a partir de dos referentes distintos: a) firmas con tecnologías homogéneas y b) empresas con tecnologías heterogéneas. Se parte de una función inversa de la demanda lineal y se asumen estructuras de costos diferentes para las empresas, en cada uno de los escenarios.

Cuando se realiza el análisis de cada uno de los tipos de firmas, se llega a dos resultados importantes: a) si surge una operación de integración empresarial entre empresas homogéneas, el ente integrado con la misma tecnología de las empresas iniciales presionará al alza el precio del mercado; b) en presencia de bienes homogéneos se tiende a subestimar el efecto real que una operación de integración produce en los precios cuando las firmas actúan estratégicamente.

En el escenario de firmas heterogéneas se encuentra como posible resultado un efecto al alza sobre el precio, que dependerá de las diferencias entre los costos marginales de las empresas intervinientes y la brecha existente entre el precio de reserva y el costo marginal más alto.

El artículo se divide en cinco partes: en la primera se explican las variables que van a ser utilizadas en la modelación; en la segunda se presenta la función inversa de la demanda lineal, de la que surgen los dos escenarios claves que se analizan en el documento; en la tercera se expone el modelo de firmas con tecnologías homogéneas; en la cuarta parte se presenta el modelo de firmas con tecnologías heterogéneas, y en la quinta se proponen las conclusiones.

\section{DEFINICIÓN DE VARIABLES}

A efectos de avanzar en la modelación propuesta, en la siguiente tabla se presenta el listado de variables que se utilizarán, con su respectiva definición" ${ }^{1}$.

Tabla 1.

Variables de estudio utilizadas

\begin{tabular}{|c|c|}
\hline Variable & Definición \\
\hline$N$ & Número de firmas participantes en el mercado. \\
\hline$q_{i}$ & Volumen de colocación de la firma. \\
\hline$P$ & Precio por unidad de producto. \\
\hline$Q$ & Volumen total de colocación. \\
\hline a & $\begin{array}{l}\text { Precio de reserva (disponibilidad máxima a } \\
\text { pagar por parte de los consumidores por el } \\
\text { bien o servicio). }\end{array}$ \\
\hline$b$ & $\begin{array}{l}\text { Parámetro de sensibilidad que mide el efecto } \\
\text { marginal, que sobre el precio causa un cambio } \\
\text { en el volumen de colocación en el mercado. }\end{array}$ \\
\hline$C_{T i}\left(q_{i}\right)$ & $\begin{array}{l}\text { Costo total de producción que enfrenta } \\
\text { la firma. }\end{array}$ \\
\hline$P(Q)$ & $\begin{array}{l}\text { Función inversa de demanda que representa } \\
\text { las preferencias de los consumidores. }\end{array}$ \\
\hline$S_{i}$ & $\begin{array}{c}\text { Participación, en volúmenes, de la firma en el } \\
\text { mercado. }\end{array}$ \\
\hline
\end{tabular}

\section{DEFINICIÓN DE RELACIONES FUNCIONALES EN LA MODELACIÓN}

Se asume una función inversa de la demanda lineal representada como:

$$
\begin{aligned}
& P(Q)=a-b Q \quad a, b>0 \\
& \text { Siendo } \\
& Q=\sum_{m=1}^{N} q_{m}
\end{aligned}
$$

Se parte de dos escenarios que se caracterizan por la homogeneidad $\mathrm{u}$ heterogeneidad en sus tecnologías: en el escenario 1, tecnologías homogéneas; en el escenario 2, tecnologías heterogéneas. La diferencia substancial entre ambos escenarios es la rigidez o variabilidad respectiva en

1 La notación sigue una presentación estándar que puede consultarse en Shy (1996). Para el lector desprevenido que quisiera contextualizarse sobre este tipo de modelos, ver Varian (1992) o Pindyck y Rubinfeld (2013). 
los costos marginales entre cada una de las firmas. A su vez, se asumen rendimientos constantes a escala ${ }^{2}$, lo que implica que la función de costos de la firma sea expresada en las ecuaciones [2] y [3] de la siguiente forma.

Escenario 1:

$C_{T_{i}}\left(q_{i}\right)=c q_{i} \quad a>c$

Escenario 2:

$C_{T_{i}}\left(q_{i}\right)=c_{i} q_{i} \quad a>\left\{c_{i}\right\}_{i=1}^{N}$

\section{ESCENARIO 1: MERCADO DE FIRMAS CON TECNOLOGÍAS HOMOGÉNEAS}

Un primer paso en la modelación consiste en reconocer un número finito de firmas, cuyas tecnologías pueden ser modeladas a partir de una única función de costos. De esta forma, el problema de la firma $i$ podrá expresarse del siguiente modo en la ecuación [4]:

$$
\max _{q_{i} \in[0, \infty)} a q_{i}-b q_{i}^{2}-b q_{i} \sum_{j \neq i}^{N} q_{j}-c q_{i}
$$

A partir de la ecuación [4] encontramos la condición de primer orden en la ecuación [5]:

$a-2 b q_{i}-b \sum_{j \neq i}^{N} q_{j}-c=0$

Y la función de mejor respuesta de la firma $i$ se encuentra en la ecuación [6]:

$q_{i}\left(q_{-i}\right)=\frac{a-c}{2 b}-\frac{1}{2} \sum_{j \neq i}^{N} q_{j}$

En equilibrio, el volumen de colocación de la firma $i$ será:

$q_{1}{ }^{*}=q_{2}{ }^{*}=\cdots=q_{N}{ }^{*}=q^{*}$

Lo que implica que el volumen de colocación de cada firma está expresado en la ecuación [7]:

$q^{*}=\left(\frac{a-c}{2 b}\right)-\frac{1}{2}(N-1) q^{*}$

$q^{*}\left[1+\frac{1}{2}(N-1)\right]=\frac{a-c}{2 b}$

$2 \quad$ El señalado supuesto se asume por simplicidad en la modelación, donde se reconoce a c como el nivel de costo marginal constante en un análisis de largo plazo. No obstante, futuros trabajos deberán concentrarse en entender las implicaciones de asumir otro tipo de rendimientos a escala en los resultados acá presentados.
$q^{*}=\frac{a-c}{(N+1) b}$

Considerando que $Q=\sum_{m=1}^{N} q_{m}$ y el nivel de producto de cada firma en el equilibrio, se obtiene la ecuación [8]:

$Q^{*}=\frac{N}{N+1} \frac{(a-c)}{b}$

Reemplazando la ecuación [8] en la función inversa de la demanda [1], obtenemos:

$P^{*}=\left(\frac{1}{N+1}\right) a+\left(\frac{N}{N+1}\right) c$

Obsérvese que el precio que se obtiene en equilibrio corresponde a un promedio ponderado entre el precio de reserva y el costo marginal, cuyos ponderadores dependen del número de firmas presentes en el mercado. Por último, los beneficios de cada una de las firmas en el equilibrio estarán dadas por la ecuación [10]:

$\pi^{*}=\frac{(a-c)^{2}}{(N+1)^{2} b}$

Mediante un ejercicio de estática comparativa (tabla 2 ), es posible cuantificar el efecto que causaría en el mercado una reducción en el número de firmas participantes, producto de una integración empresarial.

Tabla 2.

Síntesis de resultados del modelo

\begin{tabular}{|c|c|}
\hline $\begin{array}{c}\text { Variable } \\
(V)\end{array}$ & $\begin{array}{c}\text { Cambio de la variable con respecto a } \\
\left(\frac{\partial V}{\partial N}\right) \text { y signo }\end{array}$ \\
\hline$q^{*}$ & $\frac{c-a}{(N+1)^{2} b}<0$ \\
\hline$Q^{*}$ & $\frac{1}{(N+1)^{2}} \frac{a-c}{b}>0$ \\
\hline$P^{*}$ & $\frac{c-a}{(N+1)^{2}}<0$ \\
\hline$\pi^{*}$ & $-\frac{2(a-c)^{2}}{(N+1)^{3} b}<0$ \\
\hline
\end{tabular}

Fuente: elaboración de los autores. 
A partir de la expresión que establece el precio de equilibrio, se infiere que si surge una operación de integración empresarial entre empresas homogéneas, con una sola empresa integrada con la misma tecnología de las empresas iniciales, se obtendrá como resultado un diferencial de precios positivo igual a:

$p^{f}-p^{i}=\left(\frac{k}{(N-k+1)(N+1)}\right)(a-c)>0$

Donde $p^{f}$ y $p^{i}$ son el precio pos-y preintegración, respectivamente. De lo anterior se concluye que una operación de integración empresarial de tipo horizontal, entre $k$ firmas, que produce un bien homogéneo y es producida por firmas con tecnologías idénticas, generará como resultado un incremento en el precio del mercado.

De igual manera, puede calcularse la participación que cada una de las firmas tendría en el equilibrio de la siguiente forma:

$s_{i}=\frac{q_{i}^{*}}{Q^{*}}=\frac{1}{N}$

Así, de llevarse a cabo una operación de integración empresarial entre un grupo de $\$ k \$$ firmas participantes en el mercado, la participación resultante de los nuevos incumbentes será:

$s_{i}=\frac{1}{(N-k+1)}$

Es importante resaltar que la participación en mención del ente integrado no corresponde a la agregación de las participaciones de cada una de las firmas, como convencionalmente se suele asumir en un análisis de este tipo de operación; es decir, $\frac{k}{N}$ y esta última medida estará siempre por encima de la participación que se derivaría del equilibrio Nash Cournot. De lo anterior se concluye que, en presencia de bienes homogéneos, la participación del ente integrado es igual a la suma de las participaciones de las intervinientes, que implica sobreestimar la participación de dicha empresa si existe un comportamiento estratégico por parte de cada una de las empresas. $\mathrm{O}$ lo que es equivalente, se tiende a subestimar el efecto real que una operación de integración produce en los precios cuando las firmas actúan estratégicamente:

$$
\begin{aligned}
& \frac{1}{N-k+1}<\frac{k}{N} \\
& N(1-k)<k(1-k) \\
& N>k
\end{aligned}
$$

\section{ESCENARIO 2: MERCADO DE FIRMAS CON TECNOLOGÍAS HETEROGÉNEAS}

Después de haber analizado el caso de la homogeneidad entre las tecnologías de las firmas, resulta importante indagar el efecto que sobre el precio se produce cuando interactúan en una estructura oligopólica ciertas firmas cuyos costos y tecnologías resultan disímiles. En este caso, el problema de la firma $i$ será:

$\max _{q_{i} \in[0, \infty)} a q_{i}-b q_{i}^{2}-b q_{i} \sum_{j \neq i}^{N} q_{j}-c_{i} q_{i}$

La condición de primer orden será:

$a-2 b q_{i}-b \sum_{j \neq i}^{N} q_{j}-c_{i}=0$

Y la función de mejor respuesta será la siguiente:

$q_{i}\left(q_{-i}\right)=\frac{a-c_{i}}{2 b}-\frac{1}{2} \sum_{j \neq i}^{N} q_{j}$

Al reescibir esta función de mejor respuesta, es posible encontrar que el volumen de colocación para la firma $i$ es:

$q_{i}-\frac{1}{2} q_{i}=\frac{a-c_{i}}{2 b}-\frac{1}{2} \sum_{j=i}^{N} q_{j}$
$q_{i}=\frac{a-c_{i}}{b}-\sum_{j=i}^{N} q_{j}$

De esta manera, el volumen total de colocación en el mercado $\left(Q^{*}\right)$ será:

$Q^{*}=N\left(\frac{a}{b}\right)-\frac{1}{b} \sum_{j=1}^{N} c_{j}-N Q^{*}$
$Q^{*}=\frac{1}{(N+1) b}\left(N a-\sum_{j=1}^{N} c_{j}\right)$

El valor del precio en equilibrio $P^{*}$ será entonces:

$P^{*}=a \frac{1}{(N+1)}+\frac{1}{(N+1)} \sum_{j=1}^{N} c_{j}$ 
Por simplicidad se considerarán dos grupos de empresas: $\mathrm{H}$ firmas con costos altos $C_{H}$ y $L$ firmas con costos bajos $C_{L}$. Lo que se resume en la siguiente ecuación:

$H+L=N$

Se asume que se llevará a cabo una integración de tipo horizontal entre dos empresas: una de costos bajos y otra de costos altos. Como resultado, la firma integrada adoptará la tecnología de costos bajos. Por lo tanto, la agregación de costos unitários antes y después de la operación estará dada por:

N

$\sum_{j=1} c_{j}=H c_{H}+L c_{L}$

$\sum_{j=1}^{N-1} c_{j}=(H-1) c_{H}+L c_{L}$

Así, en el equilibrio, el precio resultante en el mercado antes y después de la integración será, respectivamente:

$P_{\text {exante }}=\frac{1}{N+1} a+\frac{H}{N+1} c_{H}+\frac{L}{N+1} c_{L}$

$P_{\text {expost }}=\frac{1}{N} a+\frac{H-1}{N} c_{H}+\frac{L}{N} c_{L}$

La diferencia entre los dos precios anteriormente descritos corresponde a la siguiente expresión:

$P_{\text {expost }}-P_{\text {exante }}=a\left(\frac{1}{N(N+1)}\right)+$

$c_{H}\left(\frac{H-(N+1)}{N(N+1)}\right)+c_{L}\left(\frac{L}{N(N+1)}\right)$

De la ecuación [25] se reconoce un umbral a partir del cual se puede inferir que la operación de integración generará un incremento, una reducción o una invarianza en el nivel de precios. En particular, de acuerdo con este modelo, se infiere una posible alza en precios siempre que suceda lo que se expone a continuación:

$$
\begin{gathered}
P_{\text {expost }}-P_{\text {exante }}>0 \\
\text { si y solo si } \\
a+L c_{L}>L c_{H}+c_{H}
\end{gathered}
$$

O equivalentemente:

$L<\frac{a-c_{H}}{c_{H}-c_{L}}$

De la ecuación [26] se infiere que para un nivel lo suficientemente reducido de firmas de costo bajo, existe riesgo de que, aun operaciones de integraciones de tipo horizontal que generen eficiencias productivas, se produzca una presión al alza en precios.

\section{CONSIDERACIONES FINALES}

El artículo ha presentado dos resultados esenciales atribuibles a mercados oligopólicos de bienes homogéneos, en los que las firmas compiten a la Cournot y las preferencias de los consumidores permiten concebir funciones inversas de demanda lineales. Se ha mostrado, a la luz de comportamientos estratégicos de las firmas, que: a) el análisis tradicional de una operación de integración horizontal puede representar una subestimación de la presión de precios resultante de un comportamiento estratégico; y b) desde la hipótesis de bienes homogéneos y estructuras de costos similares, cualquier operación de integración empresarial, que genere como resultado una empresa con tecnología similar a las empresas que inicialmente participan en la operación, conducirá a un incremento en el precio.

Por su parte, en el escenario de costos heterogéneos, se ha mostrado que el efecto de la integración de tipo horizontal, entre una empresa eficiente y otra ineficiente, en la que existe una adopción de tecnología eficiente por parte del ente integrado, producirá como resultado un efecto sobre el precio que dependerá de las diferencias entre los costos marginales de las empresas intervinientes y la brecha existente entre el precio de reserva y el costo marginal más alto. En particular, el documento ha mostrado que, para un número lo suficientemente bajo de firmas de costo bajo, la operación tenderá a generar presiones que incrementen el precio de mercado, en detrimento de los consumidores. 
Lo anterior permite reconocer teóricamente la importancia del rol del control previo de integraciones, incluso en aquellas operaciones de fusiones empresariales de tipo horizontal que puedan generar ganancias en materia eficiencia productiva, como el que se ha modelado en el escenario 2, en el que se reconoce el riesgo del efecto de explotación sobre el consumidor. Esto induce a considerar como relevante la existencia de una función ex ante de autoridades de competencia que permita proteger a los consumidores de riesgos inminentes derivados de la pérdida de competencia en los mercados.

Futuras investigaciones deberán centrarse en el efecto explotación anteriormente señalado, frente a operaciones de integración empresarial en las que los consumidores perciben diferenciación de los productos que se ofrecen en el mercado, dentro del marco acá propuesto. Igualmente importante será realizar un análisis de bienestar social de cada una de las operaciones de fusión modeladas en este artículo.

\section{REFERENCIAS}

1. Beckman S., DeAngelo G. y Smith, J. (2012). Expermients on horizontal mergers: does size matter? Econmics Letters, 117(3). Recuperado de https://ideas.repec.org/a/eee/ecolet/v117y2012i3p537-539.html

2. Bertrand, J. (1883). Théorie des Richesses: revue de Théories mathématiques de la richesse sociale, par Léon Walras et Recherches sur les principes mathématiques de la théorie des richesses par Augustin Cournot. Journal des Savants, 499-508. Recuperado de http://cruel.org/econthought/texts/marginal/ bertrand83.pdf

3. Chamberlin, E. (1933). The theory of monopolistic competition. Cambridge: Harvard University.

4. Cournot A. (1838). Researches into the mathematical principles of the theory of wealth. Londres: MacMillan.

5. Ding, W. Fan, C. y Wolfstetter E. (2013). Horizontal mergers with synergies: Cash vs Profit Share Auctions. International Journal Organization, 31(5). Recuperado de http:/www.sciencedirect.com/science/article/ pii/S016771871300074X

6. Eckbo, B. (1983). Horizontal mergers, collusion, and stockholder wealth. Journal of Financial Economics, 11, 241-273.

7. Epstein, R. y Rubinfeld, D. (2001). Merger simulation: a simplified approach with new applications. Antitrust Journal, 69, 883-919.

8. García, J. y Trillas, F. (2011). Control corporativo y riqueza de los accionistas en el sector eléctrico europeo, 2000-2007. Revista de Economía Institucional, 13(25), 297-319.

9. Pyndick, R. y Runbinfeld, D. (2013). Microeconomics (8. ${ }^{a}$ ed.). Nueva York: Pearson.

10. Shapiro C. (1996). Mergers with differentiated products. Recuperado de http://faculty.haas.berkeley.edu/ shapiro/diversion.pdf

11. Shy, O. (1996). Industrial organization: theory and applications. Cambridge: The MIT Press.

12. Tsitsiklis, J. y Xu Y. (2014). Efficiency loss in a cournot oligopoly with convex market demand. Journal of Mathematical Economics, 53. Recuperado de http://www.mit.edu/ yunjian/Cournot.pdf

13. Varian, H. (1992). Microeconomic analysis (3. a ed.). Nueva York: W. W. Norton Company.

14. Von Stackelberg, H. (1934). Market structure and equilibrium. Vienna: Springer Verlag.

15. Werden, G. (1997). Simulating the effects of differentiated products mergers: A practical alternative to structural merger policy. George Mason Law Review, 5, 363-386. 